\title{
Predicting the Response to Non-invasive Brain Stimulation in Stroke
}

\author{
Smadar Ovadia-Caro ${ }^{1,2,3 *}$, Ahmed A. Khalil 1,2,4, Bernhard Sehm ${ }^{1}$, Arno Villringer ${ }^{1,2,4,5}$, \\ Vadim V. Nikulin ${ }^{1,3,6,7}$ and Maria Nazarova ${ }^{7,8}$ \\ ${ }^{1}$ Department of Neurology, Max Planck Institute for Human Cognitive and Brain Sciences, Leipzig, Germany, ${ }^{2}$ Berlin School \\ of Mind and Brain, Humboldt-Universität zu Berlin, Berlin, Germany, ${ }^{3}$ Neurophysics Group, Department of Neurology, \\ Campus Benjamin Franklin, Charité-Universitätsmedizin Berlin, Berlin, Germany, ${ }^{4}$ Center for Stroke Research Berlin, \\ Charité-Universitätsmedizin Berlin, Berlin, Germany, ${ }^{5}$ Department of Cognitive Neurology, University Hospital Leipzig and \\ Faculty of Medicine, University of Leipzig, Leipzig, Germany, ${ }^{6}$ Bernstein Center for Computational Neuroscience, Berlin, \\ Germany, ${ }^{7}$ Center for Cognition and Decision Making, Institute for Cognitive Neuroscience, National Research University \\ Higher School of Economics, Moscow, Russia, ${ }^{8}$ Federal Center for Cerebrovascular Pathology and Stroke, The Ministry of \\ Healthcare of the Russian Federation, Federal State Budget Institution, Moscow, Russia
}

Keywords: NIBS, stroke, variability, functional connectivity, ongoing neuronal oscillations, long-range temporal correlations, fMRI, EEG

Neuromodulatory non-invasive brain stimulation (NIBS) techniques are experimental therapies for improving motor function after stroke. The aim of neuromodulation is to enhance adaptive or suppress maladaptive processes of post-stroke reorganization.

However, results on the effectiveness of these methods, which include transcranial magnetic stimulation (TMS) and transcranial direct current stimulation (tDCS), are mixed. The results of recent large clinical trials and meta-analyses range from no improvement in motor function $(1,2)$ to moderate improvement $(1-6)$ at the group level. Though evidence supporting efficacy is better for TMS (7) than for tDCS (6), individual stroke patients' response to NIBS is nevertheless extremely variable (8-11). This is reminiscent of the development of other stroke therapies, such as thrombolysis and mechanical thrombectomy, where early studies were largely mixed before patient selection was refined $(12,13)$. NIBS in stroke faces a similar challenge of refining patient selection and individualizing protocols to determine its therapeutic potential.

The variable response to NIBS in stroke patients is a byproduct of multiple factors that influence response to NIBS in healthy controls $(14,15)$, as well as factors that influence the response specifically in stroke patients (8). The former include factors such as age, gender, anatomical variability, intake of stimulant substances, and baseline neurophysiological state but also technical factors such as stimulation intensity, TMS coil orientation, and stimulation duration (16-18). Specifically in stroke patients, symptom severity, size and location of lesions, stroke etiology, and time from symptom onset to intervention influence the response to NIBS as well. Importantly, these different variability-causing factors interact to affect the response to NIBS, such as the potential amplification of inter-individual differences in brain anatomy $(19,20)$ by stroke lesions $(21,22)$. Such interactions make understanding the causes of NIBS response variability in stroke challenging.

Although the need for individualized stimulation protocols in stroke patients is widely accepted, it is still unclear exactly how this will be achieved. At the very least, the factors influencing variability in healthy subjects should be controlled as much as possible through appropriate and careful study design (23) and checklist-based reporting of factors during data collection (24). To address the specific factors for stroke, patient selection for NIBS should be informed by pathophysiological processes. This requires that we know which processes are relevant, that we are capable of measuring them, and that we know the optimum timing and patient-related characteristics for treatment administration. 


\section{MODELS OF REORGANIZATION AS A BASIS FOR STIMULATION PROTOCOLS}

Until recently, NIBS protocols have mostly been based on the interhemispheric competition model $(25,26)$, which postulates that the unaffected hemisphere overly inhibits the affected hemisphere. Despite NIBS strategies based on this model being largely ineffective at the group level (27-30), it is still a popular approach used by several recent (9) and ongoing clinical trials. In severely affected patients in particular, the validity of this model has been questioned $(31,32)$ and an alternative, the vicariation model, suggested (33). The vicariation model postulates that the function of the unaffected hemisphere compensates for the impairment of the affected hemisphere, thereby presenting an adaptive, rather than maladaptive, process $(32,34-37)$.

These contradictory models have been unified in the bimodalbalance recovery model, taking us a step further to individualized therapy (25). This uses a metric, the "structural reserve," defined as the integrity of the white matter motor pathways, to determine whether the inter-hemispheric competition or vicariation model is applicable in a given patient. According to the model, in patients with high structural reserve, the overactivation of the unaffected hemisphere is maladaptive, while in patients with low structural reserve, this over-activation is compensatory. Supporting this model, severely affected patients, with presumably low structural reserve, have poorer outcomes when inhibitory NIBS protocols are applied to their unaffected hemispheres $(28,37)$, emphasizing the need to modify "one-sizefit-all" NIBS protocols.

However, it is yet to be resolved which clinical and imaging characteristics are appropriate proxies for structural reserve. Most evidence thus far comes from studies investigating the ability of these characteristics to predict stroke outcome. White matter integrity, quantified with the fractional anisotropy of white matter tracts on diffusion tensor imaging, is commonly used (38-42). However, a good predictor of stroke outcome (prognostic biomarker) is not necessarily useful for predicting the response to specific NIBS paradigms (selection biomarker) (43). Prognostic biomarkers may provide a good starting point; however, they need to be validated to demonstrate their specific role and relative importance in influencing the response to NIBS after stroke. Two recent promising studies show that behavioral measures such as the Action Research Arm Test and the FuglMeyer score are predictors of the response to NIBS in correlation with white matter integrity measured using imaging $(44,45)$. These studies show that both clinical and imaging measures associated with structural reserve influence the effectiveness of facilitation of the affected hemisphere or inhibition of the unaffected hemisphere, providing direct support for the bimodalbalance recovery model, and setting the ground for future studies validating these selection biomarkers.

On the methodological level, to develop a framework to guide individualized NIBS therapy, large studies with many patients and variables must be conducted (46). The analysis of such large-volume, complex data would be suited for machine learning approaches. Considering preliminary evidence on the high correlation between clinical and imaging-based biomarkers $(44,45)$, as well as the high correlation within the different

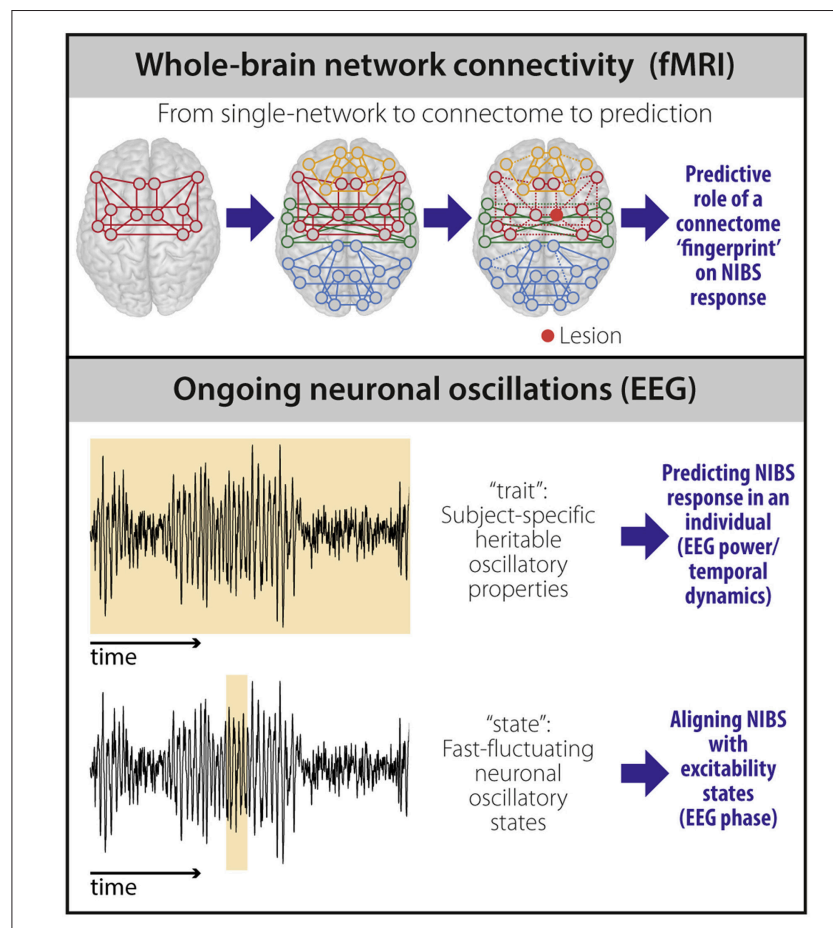

FIGURE 1 | Potential biomarkers to predict NIBS response. fMRI-based connectivity techniques (top) provide information on the brain's large-scale functional organization. Moving beyond the description of single networks, whole-brain ("connectome") connectivity models capture the heterogeneity and individual reorganization after stroke using a single scan. The individual connectome "fingerprint" could therefore be used as a predictor of NIBS response based on stroke pathophysiology in an individual patient. Properties of ongoing neuronal oscillations measured using EEG (bottom) carry both stable, heritable ("trait"), and transiently changing ("state") information. EEG power and temporal dynamics can be used as "trait" measures and provide prediction of NIBS response at the individual level. EEG phase can be used to temporally align NIBS stimulation with excitability states to improve NIBS efficacy at the individual level.

clinical features of stroke $(47,48)$, potential models guiding NIBS therapy need not to be overly complex, and it is likely that highly correlated measures can be reduced to factors of lower dimension that explain substantial variability.

Two potential imaging-based biomarkers of NIBS response in stroke-whole-brain connectivity and the brain's propensity to respond to stimulation-have been largely ignored and are addressed here (Figure 1).

\section{WHOLE-BRAIN NETWORK CONNECTIVITY}

Stroke is not a mere localized phenomenon. Widespread effects of stroke are found within the affected network (49), but also beyond it (50-54), and connectivity has been suggested as the underlying mechanism mediating these indirect effects $(33,55)$.

Whole-brain connectivity models based on resting-state functional magnetic resonance imaging (rs-fMRI) show that modulation of long-range connections between different regions outside lesions and their changes over time relate to stroke recovery on the individual level (56-58). In addition, most strokes affect multiple behavioral domains and thus changes 
in multiple functional networks better characterize a single patient. These factors likely contribute to the observed response variability to NIBS, but have not been sufficiently considered thus far, as both connectivity alterations in stroke and NIBS protocols have mostly been investigated in the context of isolated networks $(8,59-62)$. Given the effects of NIBS on distributed networks (63-65) and the understanding of stroke as a distributed pathology $(55,66,67)$, when applying stimulation in these patients, assuming that a single functional network is being, or indeed should be, targeted is problematic.

Whole-brain connectivity using rs-fMRI is well-suited for use in patients because it captures, with a single taskfree scan, information on functional connectivity of multiple brain networks $(55,66)$. In our opinion, this approach can be used to develop more realistic models of spontaneous reorganization after stroke, and could prove beneficial for designing individualized stimulation protocols.

A methodological limitation of connectivity approaches is that they rely on a-priori delineation of somewhat arbitrary boundaries between networks. Dimensionality reduction of whole-brain connections overcomes this problem (68). Using this data-driven approach, areas are clustered according to similarity of their connectivity patterns in a parametric, continuous manner. Dimensionality reduction of whole-brain connections can provide a fingerprint of the connectome at the individual patient level (69), thereby representing a more realistic picture of stroke involving multiple functional domains. Using this approach, we recently showed that the location of a stroke lesion in whole-brain connectivity space is related to the degree of reorganization that occurs within the first week of stroke onset, as measured by whole-brain functional connectivity (70). This preliminary result supports the value of developing whole-brain connectivity models to characterize the widespread effects of localized lesions in detail.

Given the promising results of predicting NIBS response using electroencephalogram (EEG) connectivity (71) and the added value of functional connectivity changes to prognostic models of stroke outcome (72), we suggest that connectivity patterns may be useful biomarkers for response to NIBS. Going forward, the link between a connectome fingerprint and spontaneous recovery in multiple functional domains has to be established, followed by the predictive role of the connectome fingerprint prior to stimulation on the clinical response to NIBS, with the eventual goal of using this information to design NIBS protocols.

\section{ONGOING NEURONAL OSCILLATIONS}

Factors influencing response to NIBS can be subclassified into momentary ("state") and phenotypic ("trait") factors. Both can be assessed using properties of neuronal oscillations that reflect the cortex's susceptibility to stimulation.

An individual's response to a stimulation protocol is hard to predict. The exact same NIBS protocol may lead to excitatory, inhibitory, or no effects on motor evoked potentials in different individuals, even in the absence of pathology $(14,15,73,74)$. One way to reduce this variability is to align the stimulation with states in which the brain is most susceptible ("excitability states") (75). There is evidence for the relevance of these states, including the observation that the variability of pre-stimulus alpha oscillations correlate with the variability of responses to TMS (76), power of sensorimotor mu (8-12 Hz oscillations above central-parietal electrodes) correlates with amplitude of motor evoked potentials (77), and synchronicity of mu oscillations in bilateral M1 is associated with stronger interhemispheric inhibition (75). These approaches are currently being pursued for targeted "state-dependent" NIBS $(78,79)$.

Properties of neuronal oscillations define instantaneous cortical reactivity to NIBS but are also subject-specific and highly heritable. This particularly relates to the power in the alpha band (80), and the temporal dynamics of the oscillations in alpha and beta bands (81). These results support the idea that beyond momentary states, properties of neuronal oscillations during rest can also represent a phenotypic trait.

The response to NIBS itself is also highly heritable (82), and intra-subject reliability of NIBS response is relatively high in healthy individuals (15). A recent EEG study showed that the temporal dynamics in the alpha band obtained before stimulation correlates on an individual level with the response to paired-pulse TMS in healthy individuals (83). These studies provide evidence that cortical plasticity is in part genetically determined, indicating a trait-like capacity of the brain to be modulated.

Studies show that neural networks might operate at the critical state, representing a balance between excitation and inhibition which is optimal for information processing (8486). Critical states are also associated with the presence of long-range temporal correlations (LRTC) in the amplitude dynamics of neuronal oscillations (87). Given that LRTCs relate to cortical excitability (83), they are likely to be perturbed after stroke, as they are in several other neurological and psychiatric disorders (88-90). The patterns of perturbation may be linked to spontaneous recovery through reaching a compensatory state that effectively balances out the state of the network.

Trait-like properties of neuronal oscillations can be quantified using clinically accessible methods such as resting EEG. In our opinion, these may serve as potentially meaningful biomarkers for response to NIBS by accounting for variability in the cortex's susceptibility to stimulation in individual patients.

\section{NEW NIBS APPROACHES}

Recent developments in NIBS technology will likely contribute to individualized therapy. Moving beyond single-area stimulation, targeting specific muscle groups that play different roles in poststroke motor recovery (for example, finger flexors vs. extensors) will be possible using multi-locus TMS (91). This approach enables stimulation of multiple regions with high temporal precision, as it does not involve repositioning of the coil. The exact changes induced by NIBS on a sub-regional level (for example, in specific parts of the motor homunculus) can be predicted using advanced induced electrical field modeling (92, 93), further refining such targeting. Finally, deep brain structures, inaccessible using TMS and tDCS yet relevant for dexterity 
deficits and pathological synergies in stroke (94, 95), might be targeted using new non-invasive stimulation approaches such as transcranial focused ultrasound (96) or temporal interference (97). These technological advances along with the development and validation of meaningful biomarkers associated with response to NIBS can help advance the translation of NIBS while embracing the inevitable heterogeneity associated with stroke pathology.

\section{AUTHOR CONTRIBUTIONS}

SO-C, AK, MN, and VN project planning and conceptualization. $\mathrm{SO}-\mathrm{C}, \mathrm{AK}$, and $\mathrm{MN}$ literature search and manuscript writing and

\section{REFERENCES}

1. Elsner B, Kugler J, Pohl M, Mehrholz J. Transcranial direct current stimulation (tDCS) for improving function and activities of daily living in patients after stroke. Cochrane Database Syst Rev. (2013). doi: 10.1002/14651858.CD009645.pub2

2. Hao Z, Wang D, Zeng Y, Liu M. Repetitive transcranial magnetic stimulation for improving function after stroke. Cochrane Database Syst Rev. (2013). doi: 10.1002/14651858.CD008862.pub2

3. Adeyemo BO, Simis M, Macea DD, Fregni F. Systematic review of parameters of stimulation, clinical trial design characteristics, and motor outcomes in non-invasive brain stimulation in stroke. Front Psychiatry. (2012) 3:88. doi: 10.3389 /fpsyt.2012.00088

4. Hsu W-Y, Cheng C-H, Liao K-K, Lee I-H, Lin Y-Y. Effects of repetitive transcranial magnetic stimulation on motor functions in patients with stroke: a meta-analysis. Stroke. (2012) 43:1849-57. doi: 10.1161/STROKEAHA.111.649756

5. O'Brien AT, Bertolucci F, Torrealba-Acosta G, Huerta R, Fregni F, Thibaut A. Non-invasive brain stimulation for fine motor improvement after stroke: a meta-analysis. Eur J Neurol. (2018) 25:1017-26. doi: 10.1111/ene.13643

6. Elsner B, Kugler J, Pohl M, Mehrholz J. Transcranial direct current stimulation (tDCS) for improving activities of daily living, and physical and cognitive functioning, in people after stroke. Cochrane Database Syst Rev. (2016) 3:CD009645. doi: 10.1002/14651858.CD009645.pub3

7. Zhang L, Xing G, Shuai S, Guo Z, Chen H, McClure MA, et al. Lowfrequency repetitive transcranial magnetic stimulation for stroke-induced upper limb motor deficit: a meta-analysis. Neural Plast. (2017) 2017:2758097. doi: $10.1155 / 2017 / 2758097$

8. Raffin E, Hummel FC. Restoring motor functions after stroke: multiple approaches and opportunities. Neuroscientist. (2018) 24:400-16. doi: $10.1177 / 1073858417737486$

9. Harvey RL, Edwards D, Dunning K, Fregni F, Stein J, Laine J, et al. Randomized sham-controlled trial of navigated repetitive transcranial magnetic stimulation for motor recovery in stroke. Stroke. (2018) 49:2138-46. doi: 10.1161/STROKEAHA.117.020607

10. Harris-Love ML, Harrington RM. Non-invasive brain stimulation to enhance upper limb motor practice poststroke: a model for selection of cortical site. Front Neurol. (2017) 8:224. doi: 10.3389/fneur.2017.00224

11. Morishita T, Hummel FC. Non-invasive brain stimulation (NIBS) in motor recovery after stroke: concepts to increase efficacy. Curr Behav Neurosci Rep. (2017) 4:280-9. doi: 10.1007/s40473-017-0121-x

12. Röther J, Ford GA, Thijs VNS. Thrombolytics in acute ischaemic stroke: historical perspective and future opportunities. Cerebrovasc Dis. (2013) 35:313-9. doi: 10.1159/000348705

13. Smith WS, Furlan AJ. Brief history of endovascular acute ischemic stroke treatment. Stroke. (2016) 47:e23-6. doi: 10.1161/STROKEAHA.115.010863

14. Huang $\mathrm{Y}-\mathrm{Z}, \mathrm{Lu} \mathrm{M}-\mathrm{K}$, Antal A, Classen J, Nitsche M, Ziemann U, et al. Plasticity induced by non-invasive transcranial brain stimulation: a position paper. Clin Neurophysiol. (2017) 128:2318-29. doi: 10.1016/j.clinph.2017.09.007 revising. SO-C principle writing and revising. $\mathrm{VN}, \mathrm{BS}$, and $\mathrm{AV}$ manuscript revising or drafting.

\section{FUNDING}

$\mathrm{VN}$ and $\mathrm{MN}$ were supported by the HSE Basic Research Program and the Russian Academic Excellence Project 5-100.

\section{ACKNOWLEDGMENTS}

The authors would like to thank Ms. Christine Tan for her help with literature search.

15. López-Alonso V, Cheeran B, Río-Rodríguez D, Fernández-Del-Olmo M. Inter-individual variability in response to non-invasive brain stimulation paradigms. Brain Stimulat. (2014) 7:372-80. doi: 10.1016/j.brs.2014. 02.004

16. Guerra A, López-Alonso V, Cheeran B, Suppa A. Variability in noninvasive brain stimulation studies: reasons and results. Neurosci Lett. (2017). doi: 10.1016/j.neulet.2017.12.058. [Epub ahead of print].

17. Fertonani A, Miniussi C. Transcranial electrical stimulation: what we know and do not know about mechanisms. Neuroscientist. (2017) 23:109-23. doi: $10.1177 / 1073858416631966$

18. Li LM, Uehara K, Hanakawa T. The contribution of interindividual factors to variability of response in transcranial direct current stimulation studies. Front Cell Neurosci. (2015) 9:181. doi: 10.3389/fncel.2015.00181

19. Opitz A, Paulus W, Will S, Antunes A, Thielscher A. Determinants of the electric field during transcranial direct current stimulation. Neuroimage. (2015) 109:140-50. doi: 10.1016/j.neuroimage.2015.01.033

20. Conde V, Vollmann H, Sehm B, Taubert M, Villringer A, Ragert P. Cortical thickness in primary sensorimotor cortex influences the effectiveness of paired associative stimulation. Neuroimage. (2012) 60:864-70. doi: 10.1016/j.neuroimage.2012.01.052

21. Ameli M, Grefkes C, Kemper F, Riegg FP, Rehme AK, Karbe H, et al. Differential effects of high-frequency repetitive transcranial magnetic stimulation over ipsilesional primary motor cortex in cortical and subcortical middle cerebral artery stroke. Ann Neurol. (2009) 66:298-309. doi: 10.1002/ana.21725

22. Minjoli S, Saturnino GB, Blicher JU, Stagg CJ, Siebner HR, Antunes A, et al. The impact of large structural brain changes in chronic stroke patients on the electric field caused by transcranial brain stimulation. Neuroimage Clin. (2017) 15:106-17. doi: 10.1016/j.nicl.2017.04.014

23. Guerra A, López-Alonso V, Cheeran B, Suppa A. Solutions for managing variability in non-invasive brain stimulation studies. Neurosci Lett. (2017). doi: 10.1016/j.neulet.2017.12.060. [Epub ahead of print].

24. Chipchase L, Schabrun S, Cohen L, Hodges P, Ridding M, Rothwell J, et al. A checklist for assessing the methodological quality of studies using transcranial magnetic stimulation to study the motor system: an international consensus study. Clin Neurophysiol. (2012) 123:1698-704. doi: 10.1016/j.clinph.2012.05.003

25. Di Pino G, Pellegrino G, Assenza G, Capone F, Ferreri F, Formica D, et al. Modulation of brain plasticity in stroke: a novel model for neurorehabilitation. Nat Rev Neurol. (2014) 10:597-608. doi: 10.1038/nrneurol.2014.162

26. Fleming MK, Rothwell JC, Sztriha L, Teo JT, Newham DJ. The effect of transcranial direct current stimulation on motor sequence learning and upper limb function after stroke. Clin Neurophysiol. (2017) 128:1389-98. doi: 10.1016/j.clinph.2017.03.036

27. Malcolm MP, Triggs WJ, Light KE, Gonzalez Rothi LJ, Wu S, Reid $\mathrm{K}$, et al. Repetitive transcranial magnetic stimulation as an adjunct to constraint-induced therapy: an exploratory randomized controlled trial. Am J Phys Med Rehabil. (2007) 86:707-15. doi: 10.1097/PHM.0b013e3181 $3 \mathrm{e} 0 \mathrm{de} 0$ 
28. Ackerley SJ, Stinear CM, Barber PA, Byblow WD. Combining theta burst stimulation with training after subcortical stroke. Stroke. (2010) 41:1568-72. doi: 10.1161/STROKEAHA.110.583278

29. Talelli P, Wallace A, Dileone M, Hoad D, Cheeran B, Oliver R, et al. Theta burst stimulation in the rehabilitation of the upper limb: a semirandomized, placebo-controlled trial in chronic stroke patients. Neurorehabil Neural Repair. (2012) 26:976-87. doi: 10.1177/1545968312437940

30. Hesse S, Waldner A, Mehrholz J, Tomelleri C, Pohl M, Werner C. Combined transcranial direct current stimulation and robot-assisted arm training in subacute stroke patients: an exploratory, randomized multicenter trial. Neurorehabil Neural Repair. (2011) 25:838-46. doi: 10.1177/1545968311413906

31. Bestmann S, Swayne O, Blankenburg F, Ruff CC, Teo J, Weiskopf N, et al. The role of contralesional dorsal premotor cortex after stroke as studied with concurrent TMS-fMRI. J Neurosci. (2010) 30:11926-37. doi: 10.1523/JNEUROSCI.5642-09.2010

32. Johansen-Berg H, Rushworth MFS, Bogdanovic MD, Kischka U, Wimalaratna S, Matthews PM. The role of ipsilateral premotor cortex in hand movement after stroke. Proc Natl Acad Sci USA. (2002) 99:14518-23. doi: 10.1073/pnas.222536799

33. Carrera E, Tononi G. Diaschisis: past, present, future. Brain. (2014) 137(Pt 9):2408-22. doi: 10.1093/brain/awu101

34. Riecker A, Gröschel K, Ackermann H, Schnaudigel S, Kassubek J, Kastrup A. The role of the unaffected hemisphere in motor recovery after stroke. Hum Brain Mapp. (2010) 31:1017-29. doi: 10.1002/hbm.20914

35. Rehme AK, Fink GR, von Cramon DY, Grefkes C. The role of the contralesional motor cortex for motor recovery in the early days after stroke assessed with longitudinal FMRI. Cereb Cortex. (2011) 21:756-68. doi: 10.1093/cercor/bhq140

36. Bajaj S, Housley SN, Wu D, Dhamala M, James GA, Butler AJ. Dominance of the unaffected hemisphere motor network and its role in the behavior of chronic stroke survivors. Front Hum Neurosci. (2016) 10:650. doi: 10.3389/fnhum.2016.00650

37. Bradnam LV, Stinear CM, Barber PA, Byblow WD. Contralesional hemisphere control of the proximal paretic upper limb following stroke. Cereb Cortex. (2012) 22:2662-71. doi: 10.1093/cercor/bhr344

38. Stinear CM, Ward NS. How useful is imaging in predicting outcomes in stroke rehabilitation? Int J Stroke. (2013) 8:33-7. doi: 10.1111/j.1747-4949.2012.00970.x

39. Stinear CM, Barber PA, Petoe M, Anwar S, Byblow WD. The PREP algorithm predicts potential for upper limb recovery after stroke. Brain. (2012) $135(\mathrm{Pt}$ 8):2527-35. doi: 10.1093/brain/aws146

40. Stinear CM, Barber PA, Smale PR, Coxon JP, Fleming MK, Byblow WD. Functional potential in chronic stroke patients depends on corticospinal tract integrity. Brain. (2007) 130(Pt 1):170-80. doi: 10.1093/brain/awl333

41. Stinear CM. Prediction of motor recovery after stroke: advances in biomarkers. Lancet Neurol. (2017) 16:826-36. doi: 10.1016/S1474-4422(17)30283-1

42. Kulikova SP, Nikulin VV, Dobrynina LA, Nazarova MA. A Possible sensory interpretation of alternate motor fibers relating to structural reserve during stroke recovery. Front Neurol. (2017) 8:355. doi: 10.3389/fneur.2017.00355

43. Kidwell CS. MRI biomarkers in acute ischemic stroke: a conceptual framework and historical analysis. Stroke. (2013) 44:570-8. doi: 10.1161/STROKEAHA.111.626093

44. McCambridge AB, Stinear JW, Byblow WD. Revisiting interhemispheric imbalance in chronic stroke: a tDCS study. Clin Neurophysiol. (2018) 129:42-50. doi: 10.1016/j.clinph.2017.10.016

45. Sankarasubramanian V, Machado AG, Conforto AB, Potter-Baker KA, Cunningham DA, Varnerin NM, et al. Inhibition versus facilitation of contralesional motor cortices in stroke: Deriving a model to tailor brain stimulation. Clin Neurophysiol. (2017) 128:892-902. doi: 10.1016/j.clinph.2017.03.030

46. Hinman JD, Rost NS, Leung TW, Montaner J, Muir KW, Brown S, et al. Principles of precision medicine in stroke. J Neurol Neurosurg Psychiatry. (2017) 88:54-61. doi: 10.1136/jnnp-2016-314587

47. Corbetta M, Siegel JS, Shulman GL. On the low dimensionality of behavioral deficits and alterations of brain network connectivity after focal injury. Cortex. (2018) 107:229-37. doi: 10.1016/j.cortex.2017.12.017
48. Stinear CM, Byblow WD, Ackerley SJ, Smith M-C, Borges VM, Barber PA. PREP2: a biomarker-based algorithm for predicting upper limb function after stroke. Ann Clin Transl Neurol. (2017) 4:811-20. doi: 10.1002/acn3.488

49. Grefkes C, Ward NS. Cortical reorganization after stroke: how much and how functional? Neuroscientist. (2014) 20:56-70. doi: 10.1177/1073858413491147

50. van den Heuvel MP, Sporns O. Network hubs in the human brain. Trends Cogn Sci. (2013) 17:683-96. doi: 10.1016/j.tics.2013.09.012

51. Alstott J, Breakspear M, Hagmann P, Cammoun L, Sporns O. Modeling the impact of lesions in the human brain. PLoS Comput Biol. (2009) 5:e1000408. doi: 10.1371/journal.pcbi.1000408

52. Aerts H, Fias W, Caeyenberghs K, Marinazzo D. Brain networks under attack: robustness properties and the impact of lesions. Brain. (2016) 139(Pt 12):3063-83. doi: 10.1093/brain/aww194

53. Honey CJ, Sporns O. Dynamical consequences of lesions in cortical networks. Hum Brain Mapp. (2008) 29:802-9. doi: 10.1002/hbm.20579

54. Gratton C, Nomura EM, Pérez F, D’Esposito M. Focal brain lesions to critical locations cause widespread disruption of the modular organization of the brain. J Cogn Neurosci. (2012) 24:1275-85. doi: 10.1162/jocn_a_00222

55. Carter AR, Shulman GL, Corbetta M. Why use a connectivity-based approach to study stroke and recovery of function? Neuroimage. (2012) 62:2271-80. doi: 10.1016/j.neuroimage.2012.02.070

56. Ramsey LE, Siegel JS, Baldassarre A, Metcalf NV, Zinn K, Shulman GL, et al. Normalization of network connectivity in hemispatial neglect recovery. Ann Neurol. (2016) 80:127-41. doi: 10.1002/ana.24690

57. Siegel JS, Seitzman BA, Ramsey LE, Ortega M, Gordon EM, Dosenbach NUF, et al. Re-emergence of modular brain networks in stroke recovery. Cortex. (2018) 101:44-59. doi: 10.1016/j.cortex.2017.12.019

58. Ovadia-Caro S, Villringer K, Fiebach J, Jungehulsing GJ, van der Meer E, Margulies DS, et al. Longitudinal effects of lesions on functional networks after stroke. J Cereb Blood Flow Metab. (2013) 33:1279-85. doi: $10.1038 / \mathrm{jcbfm} .2013 .80$

59. Volz LJ, Rehme AK, Michely J, Nettekoven C, Eickhoff SB, Fink GR, et al. Shaping early reorganization of neural networks promotes motor function after stroke. Cereb Cortex. (2016) 26:2882-94. doi: 10.1093/cercor/bhw034

60. Li LM, Violante IR, Leech R, Hampshire A, Opitz A, McArthur D, et al. Cognitive enhancement with Salience Network electrical stimulation is influenced by network structural connectivity. Neuroimage. (2019) 185:425-33. doi: 10.1016/j.neuroimage.2018.10.069

61. Li LM, Violante IR, Leech R, Ross E, Hampshire A, Opitz A, et al. Brain state and polarity dependent modulation of brain networks by transcranial direct current stimulation. Hum Brain Mapp. (2019) 40:904-15. doi: $10.1002 / \mathrm{hbm} .24420$

62. Violante IR, Li LM, Carmichael DW, Lorenz R, Leech R, Hampshire A, et al. Externally induced frontoparietal synchronization modulates network dynamics and enhances working memory performance. Elife. (2017) 6:e22001 doi: 10.7554/eLife. 22001

63. Sehm B, Schäfer A, Kipping J, Margulies D, Conde V, Taubert M, et al. Dynamic modulation of intrinsic functional connectivity by transcranial direct current stimulation. J Neurophysiol. (2012) 108:3253-63. doi: 10.1152/jn.00606.2012

64. Dijkhuizen RM, Zaharchuk G, Otte WM. Assessment and modulation of resting-state neural networks after stroke. Curr Opin Neurol. (2014) 27:637-43. doi: 10.1097/WCO.0000000000000150

65. Antonenko D, Schubert F, Bohm F, Ittermann B, Aydin S, Hayek $\mathrm{D}$, et al. tDCS-induced modulation of GABA levels and resting-state functional connectivity in older adults. J Neurosci. (2017) 37:4065-73. doi: 10.1523/JNEUROSCI.0079-17.2017

66. Ovadia-Caro S, Margulies DS, Villringer A. The value of resting-state functional magnetic resonance imaging in stroke. Stroke. (2014) 45:2818-24. doi: 10.1161/STROKEAHA.114.003689

67. Siegel JS, Ramsey LE, Snyder AZ, Metcalf NV, Chacko RV, Weinberger $\mathrm{K}$, et al. Disruptions of network connectivity predict impairment in multiple behavioral domains after stroke. Proc Natl Acad Sci USA. (2016) 113:E4367-76. doi: 10.1073/pnas.1521083113

68. Margulies DS, Ghosh SS, Goulas A, Falkiewicz M, Huntenburg JM, Langs $\mathrm{G}$, et al. Situating the default-mode network along a principal gradient of macroscale cortical organization. Proc Natl Acad Sci USA. (2016) 113:12574-9. doi: $10.1073 /$ pnas. 1608282113 
69. Finn ES, Shen X, Scheinost D, Rosenberg MD, Huang J, Chun MM, et al. Functional connectome fingerprinting: identifying individuals using patterns of brain connectivity. Nat Neurosci. (2015) 18:1664-71. doi: 10.1038/nn.4135

70. Bayrak S, Khalil AA, Villringer K, Fiebach JB, Villringer A, Margulies DS, et al. The impact of ischemic stroke on connectivity gradients: BioRxiv. (2018). doi: $10.1101 / 481689$

71. Hordacre B, Moezzi B, Goldsworthy MR, Rogasch NC, Graetz LJ, Ridding MC. Resting state functional connectivity measures correlate with the response to anodal transcranial direct current stimulation. Eur J Neurosci. (2017) 45:837-45. doi: 10.1111/ejn.13508

72. Puig J, Blasco G, Alberich-Bayarri A, Schlaug G, Deco G, Biarnes $\mathrm{C}$, et al. Resting-state functional connectivity magnetic resonance imaging and outcome after acute stroke. Stroke. (2018) 49:2353-60. doi: 10.1161/STROKEAHA.118.021319

73. Hamada M, Murase N, Hasan A, Balaratnam M, Rothwell JC. The role of interneuron networks in driving human motor cortical plasticity. Cereb Cortex. (2013) 23:1593-605. doi: 10.1093/cercor/bhs147

74. Müller-Dahlhaus JFM, Orekhov Y, Liu Y, Ziemann U. Interindividual variability and age-dependency of motor cortical plasticity induced by paired associative stimulation. Exp Brain Res. (2008) 187:467-75. doi: 10.1007/s00221-008-1319-7

75. Stefanou M-I, Desideri D, Belardinelli P, Zrenner C, Ziemann U. Phase synchronicity of $\mu$-rhythm determines efficacy of interhemispheric communication between human motor cortices. J Neurosci. (2018) 38:1052534. doi: 10.1523/JNEUROSCI.1470-18.2018

76. Iscan Z, Nazarova M, Fedele T, Blagovechtchenski E, Nikulin VV. Prestimulus Alpha oscillations and inter-subject variability of motor evoked potentials in single- and paired-pulse TMS paradigms. Front Hum Neurosci. (2016) 10:504. doi: 10.3389/fnhum.2016.00504

77. Thies M, Zrenner C, Ziemann U, Bergmann TO. Sensorimotor mu-alpha power is positively related to corticospinal excitability. Brain Stimulat. (2018) 11:1119-22. doi: 10.1016/j.brs.2018.06.006

78. Bergmann TO. Brain state-dependent brain stimulation. Front Psychol. (2018) 9:2108. doi: 10.3389/fpsyg.2018.02108

79. Thut G, Bergmann TO, Fröhlich F, Soekadar SR, Brittain J-S, ValeroCabré A, et al. Guiding transcranial brain stimulation by EEG/MEG to interact with ongoing brain activity and associated functions: a position paper. Clin Neurophysiol. (2017) 128:843-57. doi: 10.1016/j.clinph.2017. 01.003

80. Smit DJA, Posthuma D, Boomsma DI, Geus EJC. Heritability of background EEG across the power spectrum. Psychophysiology. (2005) 42:691-7. doi: 10.1111/j.1469-8986.2005.00352.x

81. Linkenkaer-Hansen K, Smit DJA, Barkil A, van Beijsterveldt TEM, Brussaard AB, Boomsma DI, et al. Genetic contributions to long-range temporal correlations in ongoing oscillations. J Neurosci. (2007) 27:13882-9. doi: 10.1523/JNEUROSCI.3083-07.2007

82. Missitzi J, Gentner R, Geladas N, Politis P, Karandreas N, Classen J, et al. Plasticity in human motor cortex is in part genetically determined. J Physiol. (2011) 589(Pt 2):297-306. doi: 10.1113/jphysiol.2010.2 00600

83. Fedele $T$, Blagovechtchenski E, Nazarova M, Iscan Z, Moiseeva V, Nikulin VV. Long-range temporal correlations in the amplitude of alpha oscillations predict and reflect strength of intracortical facilitation: combined TMS and EEG study. Neuroscience. (2016) 331:109-19. doi: 10.1016/j.neuroscience.2016.06.015

84. Shew WL, Plenz D. The functional benefits of criticality in the cortex. Neuroscientist. (2013) 19:88-100. doi: 10.1177/1073858412445487
85. Deco G, Jirsa VK. Ongoing cortical activity at rest: criticality, multistability, and ghost attractors. J Neurosci. (2012) 32:3366-75. doi: 10.1523/JNEUROSCI.2523-11.2012

86. Shew WL, Yang H, Petermann T, Roy R, Plenz D. Neuronal avalanches imply maximum dynamic range in cortical networks at criticality. J Neurosci. (2009) 29:15595-600. doi: 10.1523/JNEUROSCI.3864-09.2009

87. Poil S-S, Hardstone R, Mansvelder HD, Linkenkaer-Hansen K. Criticalstate dynamics of avalanches and oscillations jointly emerge from balanced excitation/inhibition in neuronal networks. J Neurosci. (2012) 32:9817-23. doi: 10.1523/JNEUROSCI.5990-11.2012

88. Nikulin VV, Jönsson EG, Brismar T. Attenuation of long-range temporal correlations in the amplitude dynamics of alpha and beta neuronal oscillations in patients with schizophrenia. Neuroimage. (2012) 61:162-9. doi: 10.1016/j.neuroimage.2012.03.008

89. Montez T, Poil S-S, Jones BF, Manshanden I, Verbunt JPA, van Dijk BW, et al. Altered temporal correlations in parietal alpha and prefrontal theta oscillations in early-stage Alzheimer disease. Proc Natl Acad Sci USA. (2009) 106:1614-9. doi: 10.1073/pnas.0811699106

90. Hohlefeld FU, Huebl J, Huchzermeyer C, Schneider GH, Schönecker T, Kühn $\mathrm{AA}$, et al. Long-range temporal correlations in the subthalamic nucleus of patients with Parkinson's disease. Eur J Neurosci. (2012) 36:2812-21. doi: 10.1111/j.1460-9568.2012.08198.x

91. Koponen LM, Nieminen JO, Ilmoniemi RJ. Multi-locus transcranial magnetic stimulation-theory and implementation. Brain Stimulat. (2018) 11:849-55. doi: 10.1016/j.brs.2018.03.014

92. Salo KS-T, Vaalto SM, Mutanen TP, Stenroos M, Ilmoniemi RJ. Individual activation patterns after the stimulation of different motor areas-a TMS-EEG study. Brain Connect. (2018) 8:420-8. doi: 10.1089/brain.2018.0593

93. Kalloch B, Bode J, Kozlov M, Pampel A, Hlawitschka M, Sehm B, et al. Semi-automated generation of individual computational models of the human head and torso from MR images. Magn Reson Med. (2018) 81:2090-105. doi: $10.1002 / \mathrm{mrm} .27508$

94. Zaaimi B, Dean LR, Baker SN. Different contributions of primary motor cortex, reticular formation, and spinal cord to fractionated muscle activation. J Neurophysiol. (2018) 119:235-50. doi: 10.1152/jn.00672.2017

95. McPherson JG, Ellis MD, Harden RN, Carmona C, Drogos JM, Heckman CJ, et al. Neuromodulatory inputs to motoneurons contribute to the loss of independent joint control in chronic moderate to severe hemiparetic stroke. Front Neurol. (2018) 9:470. doi: 10.3389/fneur.2018.00470

96. Fini M, Tyler WJ. Transcranial focused ultrasound: a new tool for non-invasive neuromodulation. Int Rev Psychiatry. (2017) 29:168-77. doi: 10.1080/09540261.2017.1302924

97. Grossman N, Bono D, Dedic N, Kodandaramaiah SB, Rudenko A, Suk H-J, et al. Noninvasive deep brain stimulation via temporally interfering electric fields. Cell. (2017) 169:1029-41.e16. doi: 10.1016/j.cell.2017.05.024

Conflict of Interest Statement: The authors declare that the research was conducted in the absence of any commercial or financial relationships that could be construed as a potential conflict of interest.

Copyright (C) 2019 Ovadia-Caro, Khalil, Sehm, Villringer, Nikulin and Nazarova. This is an open-access article distributed under the terms of the Creative Commons Attribution License (CC BY). The use, distribution or reproduction in other forums is permitted, provided the original author(s) and the copyright owner(s) are credited and that the original publication in this journal is cited, in accordance with accepted academic practice. No use, distribution or reproduction is permitted which does not comply with these terms. 